\title{
Diagnostic Accuracy of Serum Calcitonin Gene-Related Peptide and Apolipoprotein E in Migraine: A Preliminary Study
}

This article was published in the following Dove Press journal: International Journal of General Medicine

\author{
Rabia Latif $\mathbb{D}^{\prime}$ \\ Nazish Rafique (ID) \\ Lubna Al Asoom (D) \\ Ahmed A Alsunni $\mathbb{( D}^{\prime}$ \\ Ayad Mohammed Salem (D) \\ Dinah Abdulhadi AINoaimi ${ }^{2}$ \\ Wasan Mohammed \\ AlHarbi $^{2}$ \\ Ahad Yasir Shaikh ${ }^{2}$ \\ Shoug Abdullah AlRaddadi ${ }^{2}$ \\ Leena Abdulrahman AlMuhaish ${ }^{2}$ \\ Dana Almohazey (D) $^{3}$ \\ Sayed Abdulazeez $(\mathbb{D})^{4}$ \\ J Francis Borgio ${ }^{4}$ \\ 'Department of Physiology, College of \\ Medicine, Imam Abdulrahman Bin Faisal \\ University, Dammam, Saudi Arabia; \\ ${ }^{2}$ College of Medicine, Imam Abdulrahman \\ Bin Faisal University, Dammam, Saudi \\ Arabia; ${ }^{3}$ Department of Stem Cell \\ Research, Institute for Research and \\ Medical Consultations (IRMC), Imam \\ Abdulrahman Bin Faisal University, \\ Dammam, Saudi Arabia; ${ }^{4}$ Department of \\ Genetic Research, Institute for Research \\ and Medical Consultations (IRMC), Imam \\ Abdulrahman Bin Faisal University, \\ Dammam, Saudi Arabia
}

Background: A reliable, migraine-specific biomarker has not been identified so far. Calcitonin Gene-Related Peptide (CGRP) and Apolipoprotein E (ApoE) might serve as migraine biomarkers due to their roles in migraine pathophysiology. However, their diagnostic usefulness has not been explored yet. Present study explored the diagnostic accuracy of CGRP and ApoE in migraine.

Methods: A cross-sectional, case-control study was conducted from November 2019 to April 2020 at Physiology department of our university. Fourteen female migraine patients, 18-25 years old, with confirmed "Migraine" diagnosis by a neurologist, were recruited. Control group consisted of 14 age-matched healthy females with no personal/family history of migraine. Blood was drawn once from control subjects and twice from migraine patients (ictal and interictal phase). Serum CGRP and ApoE levels were assessed by ELISA. Statistical analysis involved paired $t$-test, one-way ANOVA, Receiver operating characteristic (ROC) curves and cross-tabs.

Results: ApoE (mg/dl) was higher significantly in interictal $(1.90 \pm 0.50)$ and ictal $(1.97 \pm 0.65)$ phases of migraine compared to control $(1.07 \pm 0.26)(\mathrm{p} \leq 0.001)$. ROC curves for ApoE were significant in migraine ictal vs control $(\mathrm{AUC}=0.91$, AUC 95\% CI: 0.78-1.0) and migraine interictal vs control (AUC $=0.92$, AUC 95\% CI: 0.8-1.0) subjects. ROC curve for CGRP $(\mathrm{pg} / \mathrm{mL})$ was significant in migraine ictal vs control subjects only (AUC $=0.79$, AUC 95\% CI: 0.6-0.97). Conclusion: Serum ApoE has "excellent" accuracy to diagnose migraine patients whether in ictal or interictal phase, from healthy subjects. ApoE levels of patients in these two phases of migraine are raised significantly than healthy subjects. CGRP has "fair" diagnostic accuracy to discriminate between migraine ictal phase and healthy subjects. Its levels do not differ significantly among migraine ictal, interictal phase and healthy controls.

Keywords: apolipoprotein E, calcitonin gene-related peptide, migraine, receiver operating curve, biomarkers

\section{Introduction}

Migraine is a complex neurovascular illness characterized by frequent headache attacks of severe intensity. ${ }^{1}$ It adversely affects the life quality of affected individuals and increases the economic burden on society in terms of lost workdays. ${ }^{2}$ Migraine is diagnosed clinically, based on patients' symptoms and the diagnostic criteria proposed by the International Headache Society (IHS). ${ }^{3}$ However, IHS diagnostic criteria are not recommended in patients with impaired cognition or deafness. Since it is a self-reported questionnaire, recall bias also decreases its sensitivity.
Correspondence: Rabia Latif

Department of Physiology, College of Medicine, Imam Abdulrahman Bin Faisa University, P.O. Box 1982, Dammam,

3144I, Saudi Arabia

Tel +966596212648

Fax +96638578048

Email rlhussain@iau.edu.sa
International Journal of General Medicine 2021:14 85I-856 
The discovery of "disease-specific" biomarkers has increased diagnostic accuracy and therapeutic efficacy in various diseases. However, there are no reliable biomarkers for migraine yet. This absence of migraine-specific biomarkers is a hindrance in developing more effective therapeutic/preventive measures. A variety of potential serum biomarkers has been proposed for migraine, including MicroRNAs, ${ }^{4}$ Calcitonin Gene-Related Peptide (CGRP) ${ }^{5}$ Apolipoprotein E (ApoE), ${ }^{6}$ Adiponectin, ${ }^{7,8}$ proinflammatory cytokines like IL-1, IL-6, TNF- $\alpha$, and anti-inflammatory cytokines like IL-10, ${ }^{9,10}$ Melatonin ${ }^{11}$ and Prostaglandins, ${ }^{12}$ etc. However, none of these biomarkers has received consensus or confirmation so far.

Among these biomarkers, CGRP seems to be the most likely candidate. CGRP levels were significantly raised in chronic migraine patients as compared to healthy controls. ${ }^{13}$ CGRP receptor antagonists can successfully treat acute migraine attacks. ${ }^{14}$ Monoclonal antibodies against CGRP are being used in migraine. ${ }^{15}$

ApoE is well known for its role in neurological and vascular disorders, ${ }^{16,17}$ headache ${ }^{18}$ and migraine. A metaanalysis of four studies (involving 649 migraineurs, 229 tension-type headache patients, and 975 healthy controls) concluded that ApoE4 (one of the alleles of the ApoE gene) is positively associated with headache and increases the relative risk of headache by 1.48 (95\% CI 1.16-1.90; $\mathrm{P}=0.002)$, compared to controls. ${ }^{19}$ In a recent study, increased ApoE levels were observed in migraineurs (interictal phase) when compared to controls. ${ }^{6}$

A precise biomarker is one that reflects pathophysiological process, has diagnostic and prognostic values, and guides in the therapeutic approach. Thus, by reflecting the pathophysiology and therapeutic response, CGRP promises to be superior to the rest of the biomarkers. However, diagnostic sensitivity and specificity of CGRP, as well as ApoE in migraine, has not been explored. Recognizing the value and importance of this knowledge gap, we conducted the present study to explore the diagnostic accuracy of CGRP and ApoE in two phases of migraine (ictal, interictal).

\section{Patients and Methods}

The study protocol was approved by the Institution Review Board of Imam Abdulrahman Bin Faisal University (IRB number IRB-UGS-2019-01-295). It was a cross-sectional case-control study, conducted from November 2019 to April 2020. The sample size was calculated by an online calculator. ${ }^{20}$ Setting power $(1-\beta)$ as
$95 \%$ and $\alpha$ (type 1 error) as $5 \%$, a sample size of 13 was finalized after putting the CGRP values of migraine cases $(74.90 \pm 28.29 \mathrm{ng} / \mathrm{mL})$ and healthy controls $(33.74 \pm 16.10$ $\mathrm{ng} / \mathrm{mL}$ ) from a previous study. ${ }^{13}$ To control for potential drop-outs and the inability of some migraine patients to come for blood extraction in ictal phase due to severe pain, we recruited 28 migraineurs in the beginning (more than double the calculated sample size).

Our inclusion criteria for migraine cases were female gender, 18-25 years of age, with confirmed "Migraine" diagnosis by a neurologist since at-least one year, fulfilling International Headache Society (IHS) criteria for migraine and willing for blood extraction at two times; ictal and interictal phase. The control group consisted of agematched healthy females with no personal or family history of migraine or subjective headache of any type. Our exclusion criteria were pregnancy, lactation, use of hormonal contraceptives, and serious active somatic/psychiatric illness.

A convenience sampling technique was used. All potential participants underwent an interview with the researchers during which inclusion/exclusion criteria were confirmed. If the conditions were fulfilled, subjects were recruited for the study. Written informed consent was signed by all participants.

Migraine phases were defined as following: ${ }^{21}$

1. The "Ictal" phase consisted of a typical throbbing headache lasting for 4-72 hours.

2. The "Interictal" phase was the period between two consecutive migraine attacks when the patient was without headache or other migraine symptoms.

Blood samples were obtained from an antecubital vein into EDTA-coated tubes, while subjects rested in sitting position. The blood was then allowed to clot and then centrifuged for 10 minutes at $2000 \mathrm{~g}$ to separate serum. Eppendorf tubes containing serum were labeled with numerical codes and stored at $-80^{\circ} \mathrm{C}$ until analyzed.

For enzyme-linked immunosorbent assay, commercial ELISA kits were used following manufacturers' instructions. Human apolipoprotein E ELISA Kit (EKC40681) Biomatik, having a detection range of $15.62 \mathrm{ng} / \mathrm{mL}-1000$ $\mathrm{ng} / \mathrm{mL}$, high sensitivity $(3.9 \mathrm{ng} / \mathrm{mL}$ ) was used to measure ApoE levels. Human Calcitonin Gene-Related Peptide ELISA Kit (EKC32941) Biomatik, having a detection range of $1.56 \mathrm{pg} / \mathrm{mL}-100 \mathrm{pg} / \mathrm{mL}$, high sensitivity $(<0.39$ $\mathrm{pg} / \mathrm{mL}$ ), specificity and precision (intra and inter-assay 
precision $<8 \%$ and $<10 \%$, respectively) was used to detect CGRP levels.

Biochemical assays were performed by investigators who were blind to the identity, diagnosis, and attack status of study participants. All blood samples were run in duplicate in 96-well plates for both assays. Later, the mean was calculated and considered for statistical analysis. This study was conducted in accordance with the Declaration of Helsinki.

\section{Statistical Analysis}

Data analysis was done by using SPSS version 20. The distribution of the biochemical variables was found to be "normal" by the Shapiro-Wilk test, therefore parametric tests were used for all the analyses. Data were presented as "mean \pm standard deviation". Serum CGRP and ApoE levels were compared among ictal, interictal phases of migraineurs and controls by ANOVA; and within-thegroup comparison was done by paired $t$-test. A receiver operating characteristic (ROC) analysis was conducted to explore diagnostic sensitivity and specificity of CGRP and ApoE in three different combinations a) Migraine ictal vs interictal, b) Migraine interictal vs control, c) Migraine ictal vs control. Co-ordinate points of the ROC curve were used to identify cut-off values. Positive predictive value (PPV) and negative predictive value (NPV) were calculated from the cross-tab function of SPSS. For all the performed tests, the level of significance was set at $p \leq 0.05$.

\section{Results}

We assessed peripheral CGRP and ApoE levels; twice in migraine patients (ictal and interictal phase) (mean age: $20.29 \pm 1.68$ years) and once in age-matched healthy controls (mean age: $21.43 \pm 1.83$ years). Within-the-group comparison by paired $t$-test revealed insignificant differences in both CGRP and ApoE in the interictal vs ictal phase of migraine (Table 1). ANOVA test (followed by
Table 2 Post Hoc Tukey HSD Test of Apolipoprotein E (mg/dl) Levels

\begin{tabular}{|l|l|}
\hline Groups & P value \\
\hline Migraine ictal vs interictal phase & 0.922 \\
\hline Migraine interictal phase vs control & 0.000 \\
\hline Migraine ictal phase vs control & 0.000 \\
\hline
\end{tabular}

Post Hoc Tukey's test) compared serum values in migraine ictal phase, interictal phase, and healthy controls and revealed significantly raised ApoE in migraine interictal and ictal phases compared to control $(p \leq 0.001)$ (Table 2).

ROC curve of migraine ictal vs interictal phase was insignificant for CGRP and ApoE both (Figure 1). ROC curve of migraine interictal vs control was significant for ApoE only (Figure 2). The area under the curve (AUC) was 0.920 (excellent accuracy) and AUC 95\% confidence interval (CI) was $0.8-1.0$, indicating that if ROC curve was repeated for 100 subjects, then $95 \%$ of subjects will have AUC in the range of 0.8 to 1.0, still in the range of good-excellent AUC (Table 3). PPV and NPV of ApoE were $68.8 \%$ and $90 \%$ respectively, meaning that $68.8 \%$ of the participants assessed as "Migraine interictal phase" based on ApoE levels were actually in that category; and $90 \%$ of the participants assessed as "Control" based on ApoE levels were actually in that category.

ROC curve of migraine ictal vs control was significant for CGRP and ApoE both (Figure 3). AUC for CGRP and ApoE was 0.786 and 0.908 , respectively, indicating "excellent" usefulness/accuracy of ApoE and a "fair" accuracy of CGRP (Table 3). 95\% CI for ApoE and CGRP was $0.8-1.0$ and $0.6-0.97$, respectively. PPV and NPV of ApoE were $83.3 \%$ and $85.7 \%$ respectively, meaning that $83.3 \%$ of the participants assessed as "Migraine ictal phase" based on ApoE levels were actually in that category; and $85.7 \%$ of the participants assessed as

Table I Comparison of Apolipoprotein E and Calcitonin Gene-Related Peptide Levels in Migraine Cases and Controls

\begin{tabular}{|l|l|l|l|l|}
\hline Variables & $\begin{array}{l}\text { Migraine Interictal } \\
\text { (Mean } \pm \text { SD) N=14 }\end{array}$ & $\begin{array}{l}\text { Migraine Ictal } \\
\text { (Mean } \pm \text { SD) N=14 }\end{array}$ & $\begin{array}{l}\text { Control (Mean } \pm \text { SD) } \\
\text { N=14 }\end{array}$ & $\begin{array}{l}\text { P value } \\
\text { (ANOVA) }\end{array}$ \\
\hline CGRP (pg/mL) & $3.19 \pm 3.36$ & $3.69 \pm 2.14$ & $2.02 \pm 1.07$ & 0.182 \\
\hline P value (paired $t$-test) & \multicolumn{2}{|c|}{0.686} & & 0.000 \\
\hline Apolipoprotein E (mg/dl) & $1.90 \pm 0.50$ & $1.97 \pm 0.65$ & & $1.07 \pm 0.26$ \\
\hline P value (paired $t$-test) & \multicolumn{2}{|c|}{0.772} & \\
\hline
\end{tabular}

Abbreviation: CGRP, calcitonin gene-related peptide. 


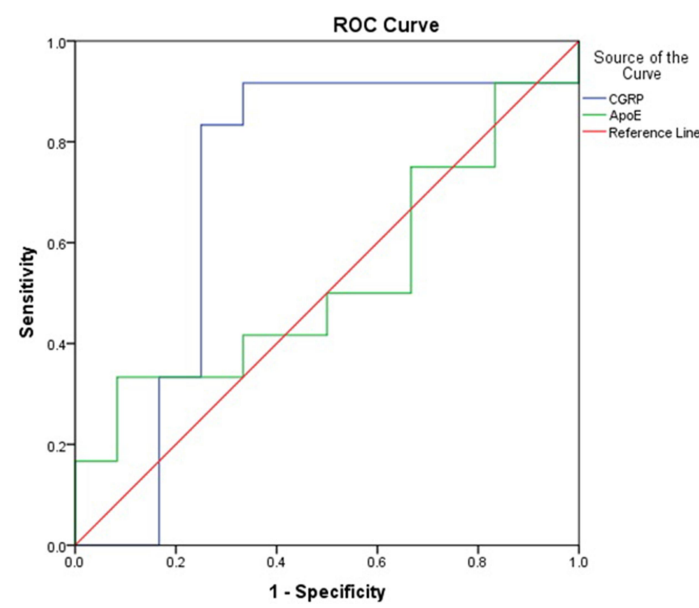

Figure I A receiver operating characteristic (ROC) curve analysis for serum apolipoprotein $E$ (ApoE) and calcitonin gene-related peptide (CGRP) in ictal vs interictal migraine phases.

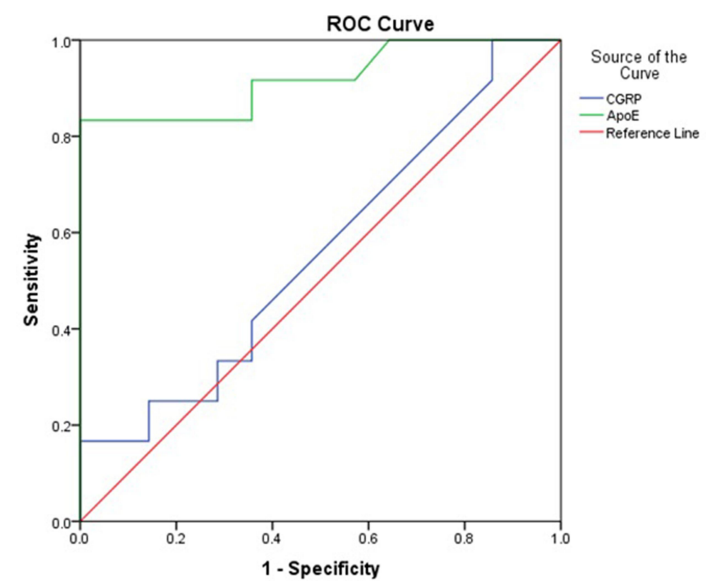

Figure 2 A receiver operating characteristic (ROC) curve analysis for serum apolipoprotein $E(A p o E)$ and calcitonin gene-related peptide (CGRP) in migraine interictal phase vs control.

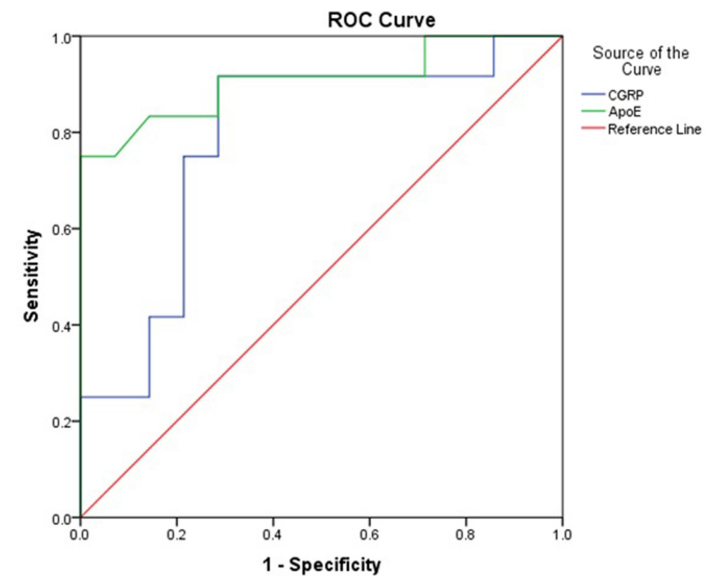

Figure 3 A receiver operating characteristic (ROC) curve analysis for serum apolipoprotein $E$ (ApoE) and calcitonin gene-related peptide (CGRP) in migraine ictal phase vs control.
"Control" based on ApoE levels were actually in that category.

\section{Discussion}

To the best of the authors' knowledge, the present study is the first one to explore diagnostic accuracy of serum ApoE and CGRP with ROC curve. According to our ROC analysis results, ApoE might be an "excellent biomarker" for diagnosis of migraine ictal vs control $(\mathrm{AUC}=0.91$, AUC 95\% CI: 0.78-1.0) and migraine interictal vs control (AUC $=0.92$, AUC 95\% CI: 0.8-1.0). We found significantly raised ApoE levels in migraine ictal and interictal phases as compared to healthy controls, a finding similar to Yuasa et al. ${ }^{6}$ ApoE increases the production of Nitric Oxide (NO) in human monocyte-derived macrophages. ${ }^{22}$ Because of the role of NO in migraine pathophysiology, ${ }^{23}$ and evidence of the higher prevalence of ApoE2 allele in migraineurs, ${ }^{24}$ ApoE levels in the blood may act as migraine biomarker.

We found insignificant changes in CGRP levels withinthe-group (migraine ictal vs interictal). In agreement with our results, Tvedskov et $\mathrm{al}^{25}$ found no increase in ictal vs interictal levels of CGRP in migraine patients; neither in external jugular vein blood samples nor in cubital vein samples. They even went to patients' residence/workplace to obtain blood samples within first 40 minutes of attack onset. In Tvedskov's study, a prolonged time delay between sampling and centrifugation might be considered a reason for little CGRP left in samples, considering the rather short half-life of CGRP. However, there was no such delay in our study. On the other hand, Gallai et $\mathrm{al}^{26}$ reported significantly raised CGRP in the ictal period compared to the interictal period in peripheral blood samples, a finding contrary to our study.

Between-the-groups comparison of interictal migraine phase vs healthy controls revealed insignificant differences in CGRP levels, a finding in agreement with Gallai et al, ${ }^{26}$ and Goadsby et $a{ }^{27}{ }^{27}$ In contrast, Cernuda-Morollón et al ${ }^{13}$ reported significantly raised CGRP levels in migraine interictal phase as compared to healthy controls; whereas $\mathrm{Han}^{5}$ reported higher CGRP levels in Migraine ictal phase than Controls. In the study conducted by Han, ${ }^{5}$ blood samples were taken from Jugular vein (vs antecubital vein in our study). Their migraine patients' mean age was $35.2 \pm 9.3$ years in contrast to $20.29 \pm 1.68$ years of mean age in our study. These variations in study methodology (i.e. differences in participants' mean age, source 
Table 3 A Receiver Operating Characteristic (ROC) Curve Analysis for Serum Apolipoprotein E and Calcitonin Gene-Related Peptide in Migraine Cases and Controls

\begin{tabular}{|c|c|c|c|c|c|c|c|c|c|}
\hline & \multirow[t]{2}{*}{ AUC } & \multicolumn{2}{|l|}{$95 \% \mathrm{Cl}$} & \multirow[t]{2}{*}{ Cutoff Points } & \multirow[t]{2}{*}{ Sen (\%) } & \multirow[t]{2}{*}{ Spe (\%) } & \multirow[t]{2}{*}{ PPV (\%) } & \multirow[t]{2}{*}{ NPV (\%) } & \multirow[t]{2}{*}{$P$ value } \\
\hline & & Lower & Upper & & & & & & \\
\hline \multicolumn{10}{|l|}{ Migraine ictal vs interictal } \\
\hline CGRP (pg/mL) & 0.708 & 0.471 & 0.946 & 2.08 & 83.3 & 75 & 76.9 & 81.8 & 0.083 \\
\hline Apolipoprotein E (mg/dl) & 0.528 & 0.287 & 0.768 & 1.59 & 75 & 33.3 & 52.9 & 57.1 & 0.817 \\
\hline \multicolumn{10}{|c|}{ Migraine interictal phase vs control } \\
\hline CGRP (pg/mL) & 0.650 & 0.334 & 0.785 & 1.59 & 41.7 & 64.3 & 44.4 & 52.9 & 0.607 \\
\hline Apolipoprotein E (mg/dl) & 0.920 & 0.804 & 1.000 & 1.20 & 91.7 & 64.3 & 68.8 & 90 & 0.000 \\
\hline \multicolumn{10}{|c|}{ Migraine ictal phase vs control } \\
\hline CGRP (pg/mL) & 0.786 & 0.599 & 0.973 & 1.88 & 91.7 & 71.4 & 73.3 & 90.9 & 0.014 \\
\hline Apolipoprotein E (mg/dl) & 0.908 & 0.782 & 1.000 & 1.39 & 83.3 & 85.7 & 83.3 & 85.7 & 0.000 \\
\hline
\end{tabular}

Abbreviations: AUC, area under curve; Cl, confidence interval; Sen, sensitivity; Spe, specificity; PPV, positive predictive value; NPV, negative predictive value; CGRP, calcitonin gene-related peptide.

of blood sample, etc.) may have contributed to results heterogeneity. ROC curve for CGRP $(\mathrm{pg} / \mathrm{mL})$ in our study was significant in migraine ictal vs control only (AUC $=0.79$, AUC 95\% CI: 0.6-0.97). Although CGRP did not emerge as significant biomarker as ApoE in our study, CGRP's role in migraine pathogenesis cannot be denied.

\section{Limitations}

Our study might be limited by a small sample size. Although we recruited 28 migraine subjects, in the beginning, we could get blood samples of 14 migraineurs only (in ictal and interictal phases) before nationwide COVID-19 strict lockdown and precautionary measures. All our subjects were females, so study findings may not be generalized. Some important information regarding migraine subjects were not recorded (e.g. duration of attack and intensity of pain at the time of ictal phase blood sampling, number of years since migraine diagnosis, family history of migraine, presence of possible pain comorbidities such as fibromyalgia, chronic pelvic pain, myofascial pain syndromes, ${ }^{28}$ etc.). In future studies, more migraineurs should be recruited. Differences in the biomarkers in various migraine subgroups such as migraineurs with and without pain comorbidities or migraineurs with or without aura should also be explored.

\section{Conclusion}

ApoE levels are raised significantly in migraine ictal and interictal phases than in healthy controls. ApoE has "excellent" accuracy to diagnose these two phases of migraine from healthy controls. CGRP levels do not differ significantly among Migraine ictal, interictal phase, and healthy controls. CGRP has "fair" accuracy to diagnose migraine ictal phase from healthy controls.

\section{Data Sharing Statement}

Data related to this manuscript can be made available from the corresponding author upon reasonable request.

\section{Acknowledgment}

The authors would like to acknowledge Ms Hind Saleh Alsuwat and Mr Ranilo M Tumbaga (Department of Genetic Research, Institute for Research and Medical Consultations (IRMC), Imam Abdulrahman Bin Faisal University) for their assistance in ELISA analysis.

\section{Disclosure}

The authors report no conflicts of interest for this work.

\section{References}

1. Hoffmann J, Baca SM, Akerman S. Neurovascular mechanisms of migraine and cluster headache. J Cereb Blood Flow Metab. 2019;39 (4):573-594. doi:10.1177/0271678X17733655 
2. Goadsby PJ, Holland PR, Martins-Oliveira M, Hoffmann J, Schankin C, Akerman S. Pathophysiology of migraine: a disorder of sensory processing. Physiol Rev. 2017;97:553-622. doi:10.1152/ physrev.00034.2015

3. Olesen J. Headache classification committee of the International Headache Society (IHS) the international classification of headache disorders, 3rd edition. Cephalalgia. 2018;38(1):1-211. doi:10.1177/ 0333102417738202

4. Gazerani P. Current evidence on potential uses of microRNA biomarkers for migraine: from diagnosis to treatment. Mol Diagn Ther. 2019;23(6):681-694. doi:10.1007/s40291-019-00428-8

5. Han D. Association of serum levels of calcitonin gene-related peptide and cytokines during migraine attacks. Ann Indian Acad Neurol. 2019;22(3):277. doi:10.4103/aian.AIAN 371 18

6. Yuasa N, Nagata E, Fujii N, Ito M, Tsukamoto H, Takizawa S. Serum apolipoprotein $\mathrm{E}$ may be a novel biomarker of migraine. PLoS One. 2018;13(1):e0190620. doi:10.1371/journal.pone.0190620

7. Duarte H, Teixeira AL, Rocha NP, Domingues RB. Increased serum levels of adiponectin in migraine. J Neurol Sci. 2014;342(1-2):186188. doi:10.1016/j.jns.2014.04.035

8. Chai NC, Gelaye B, Tietjen GE, et al. Ictal adipokines are associated with pain severity and treatment response in episodic migraine. Neurol. 2015;84:1409-1418. doi:10.1212/WNL.0000000000001443

9. Boćkowski L, Sobaniec W, Żelazowska-rutkowska B. Proinflammatory plasma cytokines in children with migraine. Pediatr Neurol. 2009;41:17-21. doi:10.1016/j.pediatrneurol.2009.02.001

10. Perini F, D'Andrea G, Galloni E, et al. Plasma cytokine levels in migraineurs and controls. Headache. 2005;45(7):926-931. doi:10. $1111 / j .1526-4610.2005 .05135 . x$

11. Masruha MR, Lin J, de Souza Vieira DS, et al. Urinary 6- sulphatoxymelatonin levels are depressed in chronic migraine and several comorbidities. Headache. 2010;50:413-419. doi:10.1111/j.15264610.2009.01547.x

12. Durham PL, Vause CV, Derosier F, McDonald S, Cady R, Martin V. Changes in salivary prostaglandin levels during menstrual migraine with associated dysmenorrhea. Headache. 2010;50(5):844-851. doi:10.1111/j.1526-4610.2010.01657.x

13. Cernuda-Morollón E, Larrosa D, Ramón C, Vega J, MartínezCamblor P, Pascual J. Interictal increase of CGRP levels in peripheral blood as a biomarker for chronic migraine. Neurology. 2013;81 (14):1191-1196. doi:10.1212/WNL.0b013e3182a6cb72

14. Mullin K, Kudrow D, Croop R, et al. Potential for treatment benefit of small molecule CGRP receptor antagonist plus monoclonal antibody in migraine therapy. Neurology. 2020;94(20):e2121-e2125. doi:10.1212/WNL.0000000000008944

15. Edvinsson L, Haanes KA, Warfvinge K, Krause DN. CGRP as the target of new migraine therapies - successful translation from bench to clinic. Nat Rev Neurol. 2018;14(6):338-350. doi:10.1038/s41582018-0003-1
16. Yamazaki Y, Zhao N, Caulfield TR, Liu C, Bu G. Apolipoprotein $\mathrm{E}$ and alzheimer disease: pathobiology and targeting strategies. Nat Rev Neurol. 2019;15:501-518.

17. Lagging C, Lorentzen E, Stanne TM, et al. Genetics of Ischaemic Stroke Functional Outcome (GISCOME) network and the international stroke genetics consortium. APOE $\varepsilon 4$ is associated with younger age at ischemic stroke onset but not with stroke outcome. Neurology. 2019;93:849-853. doi:10.1212/WNL.0000000000008459

18. Merritt VC, Ukueberuwa DM, Arnett PA. Relationship between the apolipoprotein E gene and headache following sports-related concussion. J Clin Exp Neuropsychol. 2016;38:941-949. doi:10. 1080/13803395.2016.1177491

19. Miao J, Wang F, Zheng W, Zhuang X. Association of the apolipoprotein $\mathrm{E}$ polymorphism with migraine: a meta-analysis. $B M C$ Neurol. 2015;15(1):138. doi:10.1186/s12883-015-0385-2

20. Power and Sample size. Available from: http://powerandsamplesize.com/ Calculators/Compare-2-Means/2-Sample-Equality. Accessed November, 2019.

21. Blau JN. Migraine: theories of pathogenesis. Lancet. 1992;339 (8803):1202-1207. doi:10.1016/0140-6736(92)91140-4

22. Colton CA, Brown CM, Cook D, et al. APOE and the regulation of microglial nitric oxide production: a link between genetic risk and oxidative stress. Neurobiol Aging. 2002;23(5):777-785. doi:10.1016/ s0197-4580(02)00016-7

23. Pradhan AA, Bertels Z, Akerman S. Targeted nitric oxide synthase inhibitors for migraine. Neurotherapeutics. 2018;15(2):391-401. doi:10.1007/s13311-018-0614-7

24. Gupta R, Kumar V, Luthra K, Banerjee B, Bhatia MS. Polymorphism in apolipoprotein $\mathrm{E}$ among migraineurs and tension-type headache subjects. J Headache Pain. 2009;10(2):115-120. doi:10.1007/s10194008-0094-y

25. Tvedskov JF, Lipka K, Ashina M, Iversen HK, Schiffter S, Olesen J. No increase of calcitonin-gene related peptide in jugular blood during migraine. Ann Neurol. 2005;58:561-568. doi:10.1002/ana.20605

26. Gallai V, Sarchielli P, Floridi A, et al. Vasoactive peptide levels in the plasma of young migraine patients with and without aura assessed both interictally and ictally. Cephalalgia. 1995;15:384-390. doi:10.1046/j.1468-29821995.1505384.x

27. Goadsby PJ, Edvinsson L, Ekman R. Vasoactive peptide release in the extracerebral circulation of humans during migraine headache. Ann Neurol. 1990;28(2):183-187. doi:10.1002/ana.410280213

28. Affaitati G, Costantini R, Tana C, et al. Co-occurrence of pain syndromes. J Neural Transm. 2020;127(4):625-646.
International Journal of General Medicine

\section{Publish your work in this journal}

The International Journal of General Medicine is an international, peer-reviewed open-access journal that focuses on general and internal medicine, pathogenesis, epidemiology, diagnosis, monitoring and treatment protocols. The journal is characterized by the rapid reporting of reviews, original research and clinical studies across all disease areas. The manuscript management system is completely online and includes a very quick and fair peer-review system, which is all easy to use. Visit http://www.dovepress.com/ testimonials.php to read real quotes from published authors. 